\title{
Fibrilhação auricular permanente: apresentação de dois casos clínicos
}

Daniel Collantes*

\section{RESUMO}

A fibrilhação auricular (FA) é a arritmia mais prevalente em Medicina Familiar, que afecta 1-2\% da população geral e 5\% da população com idade superior a 65 anos. Os doentes com esta arritmia têm maior risco de mortalidade e de morbilidade, nomeadamente de acidente vascular cerebral (AVC). Neste artigo procura-se reflectir sobre a aplicação das recomendações internacionais na terapêutica da FA à prática do médico de família (MF), sobretudo no que diz respeito à anticoagulação.

Para esse fim, são apresentados dois casos clínicos de doentes com FA. Em ambas as situações, eram primeiras consultas com o autor, nas quais se avaliou a adequação da terapêutica instituída e se fizeram ajustes na terapêutica da FA. Expõem-se alguns dados da literatura que serviram para fundamentar as decisões tomadas nestes dois doentes. De acordo com as normas internacionais, o controlo da frequência cardíaca associada à anti-coagulação crónica é a estratégia de actuação na maioria dos doentes com FA. Quando se pretende o controlo da frequência cardíaca, os fármacos de primeira linha são os beta-bloqueadores e os bloqueadores de canais de cálcio. Por fim, porque a anticoagulação oral não é uma terapêutica isenta de risco, existem escalas que categorizam em grupos de risco para eventos tromboembólicos. Estas escalas podem auxiliar na decisão da terapêutica anti-trombótica.

Reflecte-se ainda sobre o significado das recomendações internacionais atendendo à especificidade da medicina familiar. O MF, enquanto prestador de cuidados globais e continuados tem um papel privilegiado para aferir se, ao longo de anos de cuidados, a terapêutica da FA se mantém de acordo com a melhor evidência disponível. Por outro lado, ao conhecer o doente e o seu contexto pode, por exemplo, mobilizar a família para o controlo de uma terapêutica que precisa de uma vigilância apertada.

Palavras-Chave: Fibrilhação Auricular; Anticoagulantes; Terapêutica Farmacológica; Médicos de Família.

\section{INTRODUÇÃO}

A FA é a arritmia que mais comummente se observa na prática clínica. É mais frequente nos homens que nas mulheres e a sua prevalência aumenta com a idade, sendo de $0,5 \%$ entre os 50-59 anos, de $4 \%$ entre os 60-69 anos e de 9\% em mais de 75 anos. ${ }^{1}$ Devido à maior esperança de vida, $o$ número de pessoas atingidas está a aumentar. Aproximadamente $70 \%$ das pessoas afectadas encontramse entre os 65 e 85 anos. O estudo ATRIA (AnTicoagulation and Risk Factors in Atrial Fibrillation) demonstra que actualmente, nos Estados Unidos da América (E.U.A.), existem 2,2 milhões de pessoas com esta doença, projectando para o ano de 2050 um aumento para 5,6 milhões. ${ }^{2}$

\footnotetext{
*Assistente de Clínica Geral
}

Centro de Saúde de Portalegre
Com a apresentação destes dois casos clínicos pretende-se reflectir sobre a importância do papel da transversalidade dos cuidados prestados pelo médico de família. De facto, nos dois casos apresentados, o motivo que leva os utentes à consulta não tem relação com o problema da «fibrilhação auricular». Por outro lado, a FA permanente é um problema prevalente na comunidade e cuja gestão na prática clínica é complexa, deparando-se o médico de família com dificuldades na aplicação das recomendações.

Em concreto, com este trabalho procuram-se atingir os seguintes objectivos:

- Reavaliar a terapêutica nos casos clínicos apresentados e a sua concordância com a abordagem actualmente recomendada nos doentes com fibrilhação auricular

- Avaliar se os doentes apresentados nos dois casos 
clínicos são candidatos apropriados para a terapêutica anticoagulante, aplicando o sistema de estratificação do risco $\mathrm{CHADS}_{2}$ para identificar o seu nível de risco.

- Avaliar a compreensão do utente e o apoio familiar como factor condicionante da decisão de instaurar terapêutica anticoagulante.

\section{DESCRIÇÃO DO PRIMEIRO CASO}

Utente de sexo masculino, raça caucasiana, 73 anos de idade, aposentado, pertencente a uma família nuclear. Dos antecedentes patológicos salienta-se a existência de Hipertensão Arterial (1995), Acidente Vascular Cerebral (1999), Fibrilhação Auricular permanente (2000). Sem antecedentes familiares relevantes.

Estava medicado, desde Março de 2000, após a alta hospitalar de um internamento por AVC, com: captopril $25 \mathrm{mg}$ ( 3 por dia), diltiazem $60 \mathrm{mg}$ ( 3 por dia), amiodarona $200 \mathrm{mg}$ (1 por dia) e ácido acetilsalicílico $150 \mathrm{mg}$ (1 por dia).Em Abril de 2006 recorreu, pela primeira vez, à consulta, depois de vários anos sem ir à consulta do médico de família. Vinha acompanhado pela mulher e a filha, para se apresentar e referia queixas osteoarticulares nos joelhos. Embora o motivo da consulta fosse a patologia osteoarticular, o utente tinha antecedentes que deviam ser tidos em conta, sendo esta a primeira consulta e tendo presente a globalidade de cuidados que presta o médico de família. Assim, foram solicitadas as reavaliações electrocardiográfica e ecocardiográfica.

O electrocardiograma (ECG) mostrava bom controlo da frequência ventricular (82 b.p.m). No ecocardiograma constatou-se a presença de uma boa função sistólica (fracção de encurtamento $44 \%$ ), apesar de ter um diâmetro diastólico do ventrículo esquerdo no limite superior do normal (55 mm). A aurícula esquerda estava ligeiramente dilatada $(44 \mathrm{~mm}$.

Na consulta seguinte, em que se solicitou a presença da filha com a intenção de auxiliar no plano terapêutico, iniciou-se anticoagulação oral com varfarina $1,25 \mathrm{mg} /$ dia (1/4 cp.). Retirou-se a amiodarona e o ácido acetilsalicílico, mantendo-se o captopril e o diltiazem. Foi realizado controlo da anticoagulação no Centro de Saúde, ao fim de três dias e, após reajuste da dose de anticoagulação oral, marcou-se novo controlo ao fim de uma semana. Passadas quatro semanas conseguiu-se atingir o valor do INR entre 2-3.

\section{DESCRIÇÃO DO SEGUNDO CASO}

Utente de sexo masculino, raça caucasiana, 75 anos de idade, aposentado, activo, separado desde há seis anos, habitando sozinho.

Em Fevereiro de 2006, recorreu pela primeira vez à consulta, referindo apresentar tremor a nível das duas mãos, que lhe dificultava as actividades diárias, nomeadamente, a alimentação.

Dos antecedentes patológicos salienta-se a existência de Fibrilhação Auricular permanente (2004), em tratamento com digoxina e clopidogrel. Sem antecedentes familiares relevantes.

O último ECG (2005) prévio à consulta mostrava bom controlo da frequência ventricular (96 b.p.m). O ecocardiograma (2004) revelava uma boa função sistólica, sem hipertrofia ventricular esquerda, com a aurícula esquerda dilatada $(49 \mathrm{~mm})$. Apresentava alteração ligeira, fibrocalcificante, das válvulas aórtica e mitral. Do ponto de vista ecocardiográfico e electrocardiográfico não apresentava alterações significativas na altura da consulta (Fev. 2006).

Iniciou-se terapêutica com $40 \mathrm{mg}$ de propanolol e anticoagulação oral com varfarina, $1.25 \mathrm{mg} /$ dia (1/4 cp.). Realizou controlo da anticoagulação oral, passados 3 dias, no Centro de Saúde. Passadas 2 semanas conseguiu-se atingir o valor do INR entre 2-3.

Foram retirados a digoxina e o clopidogrel.

\section{COMENTÁRIO}

A cardiopatia hipertensiva é a causa mais frequente de FA permanente, ainda que outras condições cardíacas e não cardíacas a possam provocar (Quadro I). No entanto, entre $2-15 \%$ dos doentes que sofrem de FA não apresentam doença cardíaca estrutural: é a denominada FA solitária ou isolada (lone atrial fibrillation). ${ }^{3}$ Além disso, não devemos esquecer que nos países subdesenvolvidos a doença valvular reumática continua sendo uma causa importante de FA.

A FA tem uma apresentação clínica heterogénea e pode causar uma grande variedade de sintomas. Frequentemente leva à hospitalização por insuficiência cardíaca ou por isquémia, pode causar miocardiopatia (taquimiocardiopatia) e, talvez o mais importante, pode produzir situações trombo-embólicas. No entanto, quase $12 \%$ dos novos casos de FA são totalmente assintomáticos e diagnosticados por electrocardiogramas de 


\section{QUADRO I. Causas de fibrilhação auricular}

\section{Cardíacas}

\begin{tabular}{l|l}
\hline Hipertensão arterial & Hipertiroidismo \\
\hline Insuficiência cardíaca & Intoxicação alcoólica \\
\hline Miocardiopatia dilatada & DPOC \\
\hline Cardiopatia hipertrófica & Trombo-embolismo pulmonar \\
\hline Cardiopatia isquémica & $\begin{array}{l}\text { Autonómicas } \\
\text { (vagal e adrenérgica) }\end{array}$ \\
\hline $\begin{array}{l}\text { Calcificação do anel } \\
\text { mitral }\end{array}$ & $\begin{array}{l}\text { Fármacos(Teofilina, } \\
\text { Pós-operatório de cirurgia } \\
\text { cardiovascular }\end{array}$ \\
\hline Valvulopatia reumática & \\
\hline Cardiopatia congénita & \\
\hline Pericardite &
\end{tabular}

rotina. ${ }^{4}$ Mesmo assim, a monitorização electrocardiográfica contínua (Holter) realizada em doentes que sofreram de FA paroxística demonstra que $20 \%$ dos episódios são assintomáticos.

Existiram diferentes esquemas de classificação de FA ao longo do tempo. Actualmente, realiza-se de acordo com a sua duração e persistência. Este aspecto tem importância por condicionar o esquema terapêutico que se pode implementar. A FA aguda é definida como um episódio que dura menos de 48 horas. A FA paroxística é caracterizada por episódios intermitentes, recorrentes (mais de dois episódios) e que resolvem espontaneamente; geralmente, dura menos de 48 horas e, por definição, não deve permanecer mais de 7 dias. A FA persistente dura mais de 7 dias, sendo necessária uma intervenção terapêutica para restaurar o ritmo sinusal. A FA permanente é considerada quando o ritmo sinusal não é reinstalado depois da cardioversão. A FA crónica é um termo amplo e aplica-se quando não existe possibilidade de manter o ritmo sinusal. ${ }^{5}$

A abordagem terapêutica da FA tem tido várias mudanças ao longo do tempo. Estas mudanças incidem, essencialmente, em dois aspectos: selecção de uma estratégia de controlo de ritmo versus controlo de frequência e selecção de uma estratégia antitrombótica.

No ano 2002 foi publicado o estudo AFFIRM (Atrial Fibrillation Follow-up Investigation of Rhythm Management). ${ }^{6}$ Este era um estudo multicêntrico, controla- do e aleatorizado que envolveu 4060 utentes adultos, com uma idade média de 69,7 anos e que apresentavam FA, documentada através de registo electrocardiográfico. Este estudo concluiu que o controlo da frequência ventricular da FA e o uso da varfarina são uma alternativa terapêutica aceitável à abordagem clássica da reversão ao ritmo sinusal. Estudos posteriores como o RACE $^{7}$ (Rate Control versus Electrical Cardioversion for Persistent Atrial Fibrillation) (522 utentes) corroboraram esta conclusão.

Os resultados dos estudos AFFIRM e RACE serviram de base a recomendações de sociedades científicas como a American Academy of Family Physicians, American College of Physicians (2003) e American College of Cardiology for Practice Guidelines and Policy Conferences. ${ }^{8,9}$ Segundo estas, o controlo da frequência cardíaca associada à anti-coagulação crónica é a estratégia de actuação na maioria dos doentes com FA. A reconversão a ritmo sinusal está indicada apenas em situações particulares: doentes com sintomatologia incapacitante ou intolerância ao esforço.

Quando o objectivo terapêutico é controlar a frequência ventricular, os fármacos recomendados são: os beta-bloqueadores (atenolol, propanolol, bisoprolol, metoprolol, pindolol), os antagonistas do cálcio com efeitos inotrópicos negativos (diltiazem e verapamil) e a digoxina. Define-se como FA controlada, a que mantém entre 60 e 80 batimentos por minuto em repouso, e 90 e 115 batimentos por minuto durante o esforço moderado.

Embora tenha efeito cronotrópico negativo, a amiodarona não está recomendada para o objectivo do controlo da frequência. De facto, não acrescenta vantagens relativamente a estes grupos terapêuticos e apresenta uma alta incidência de efeitos secundários clinicamente relevantes, nomeadamente, na função tiroideia, função hepática, fibrose pulmonar e depósitos na córnea. Utiliza-se mais quando se pretende um controlo do ritmo. De facto, um dos erros mais frequentes é manter, durante anos ou de forma indefinida, o tratamento com amiodarona numa FA permanente que não se conseguiu reverter a ritmo sinusal e, muitas vezes, sem controlo da função tiroideia (que se deveria fazer de $6 \mathrm{em}$ 6 meses), função hepática e pulmonar. Num estudo prospectivo feito em 200 utentes, após 2 anos de utilização de amiodarona, na dose de $1.260 \mathrm{mg} /$ semana, $6 \%$ 
dos utentes apresentavam alterações tiroideias, $81 \%$ tinham depósitos na córnea e não houve nenhum caso de fibrose pulmonar. Em 9\% dos casos suspendeu-se o tratamento por intolerância ao medicamento. ${ }^{10}$

Os digitálicos são cada vez menos utilizados pois, apesar de controlarem a resposta ventricular em repouso, não evitam frequências elevadas durante o esforço, pelo que devem ser usados como fármacos de $2^{\mathrm{a}}$ linha, caso exista contra-indicação para o uso dos beta-bloqueadores.

Os fármacos que se utilizam para a manutenção do ritmo sinusal são os do grupo IC (propafenona, flecainida) e os do III (amiodarona e sotalol).

Existem duas estratégias de prevenção de acidentes tromboembólicos: a antiagregação e a anticoagulação. Numa revisão da Colaboração Cochrane ${ }^{11}$ foram incluídos diversos estudos, tanto em prevenção primária como secundária, da FA não valvular. Compara-se, directamente, a varfarina em doses correctas (para conseguir um INR entre 2 e 3 ) com a aspirina, e conclui-se que existe uma redução significativa do risco de AVC, três vezes superior, com varfarina. Os doentes anticoagulados tem um risco menor de AVC e de eventos cardiovasculares, mas também um acréscimo de risco de hemorragia. ${ }^{12}$ Tendo em conta esta relação de risco/benefício, para seleccionar a estratégia antitrombótica mais acertada, seria útil criar uma classificação de risco de AVC nos doentes com FA.

O AFI (Atrial Fibrillation Investigators) ${ }^{13}$ foi um estudo que reuniu 5 grandes estudos aleatorizados em doentes com FA e terapia anticoagulante. Estes investigadores realizaram uma análise multivariada para definir preditores de AVC. Esta análise demonstrou que uma variedade de factores de risco aumentavam o risco de AVC em mais de $5 \%$ por ano e a terapia anticoagulante o reduzia em aproximadamente 3 vezes. Em paralelo, os investigadores do SPAF (Stroke Prevention in Atrial Fibrillation) avaliaram o risco de apresentar um $\mathrm{AVC}^{14}$ e demonstraram resultados semelhantes (Quadro II).

No ano 2001 propôs-se um modelo de estratificação de risco denominado $\mathrm{CHADS}_{2}{ }^{15}$ (acrónimo em inglês das letras que a incluem) incorporando características do SPAF e AFI. Nesta classificação atribui-se a cada factor de risco o valor de 1 ou 2, alcançando assim num doente individual uma estratificação numérica do ris-

\begin{tabular}{|c|c|}
\hline \multicolumn{2}{|c|}{$\begin{array}{l}\text { QUADRO II. Estratificação do risco derivado dos } \\
\text { ensaios SPAF (Stroke Prevention in Atrial Fibrillation) } \\
\text { e AFI (Atrial Fibrillation Investigators) }\end{array}$} \\
\hline SPAF & AFI \\
\hline Alto risco & Alto risco \\
\hline Mulher $>75$ anos & 65 anos \\
\hline PAS $>160 \mathrm{mmHg}$ & HTA \\
\hline Disfunção ventricular esquerda & $\begin{array}{l}\text { Doença arterial coronária } \\
\text { Diabetes }\end{array}$ \\
\hline $\begin{array}{l}\text { Risco intermédio } \\
\text { HTA } \\
\text { Sem factores de alto risco }\end{array}$ & \\
\hline Baixo risco & Baixo risco \\
\hline Sem HTA & $<65$ anos \\
\hline Sem factores de alto risco & Sem factores de alto risco \\
\hline
\end{tabular}

Legenda: PAS: Pressão arterial sistólica. HTA: Hipertensão arterial.

co de AVC (Quadro III). O risco de acidente vascular cerebral associado a um valor alto de $\mathrm{CHADS}_{2}$ foi demonstrado nos E.U.A. usando um Registo Nacional de FA. ${ }^{16}$ De todas as maneiras, são necessários mais dados para indicar se este algoritmo é suficiente para a estratificação de risco ou se deverão ser incluídos outros factores. O CHADS 2 correlacionou-se muito bem com a frequência de eventos por 100 doentes/ano nos estudos AFI e SPAF. Concluiu-se ainda que os dois esquemas de classificação do risco SPAF, AFI e CHADS $_{2}$ são úteis para quantificar o risco de AVC em doentes com FA, podendo ser úteis na selecção da terapêutica antitrombótica. Numa revisão da Colaboração Cochrane ${ }^{17}$ a escala $\mathrm{CHADS}_{2}$ mostrou-se preditiva numa coorte grande de prática clínica de utentes com FA.

Em Junho de 2006, o NICE ${ }^{18}$ (Nacional Institute for Health and Clinical Excellence) publicou um guia de abordagem de FA na qual inclui uma escala nova de estratificação do risco embólico (Quadro IV). Segundo esta escala seriam de alto risco os utentes com: embolismo, AVC ou AIT prévio, valvulopatia, insuficência cardiaca ou diminuição da fracção de ejecção (FE) documentada em ecocardiograma, idade $>75$ anos com HTA, DM ou doença vascular; médio risco: > ou igual a 65 anos sem factores de risco, $<75$ anos com factores de risco (DM, HTA, vasculopatia periférica) e de baixo risco: $<65$ anos sem factores de risco. Provavelmente por 


\begin{tabular}{|l|c|}
\hline \multicolumn{2}{|l|}{ QUADRO III. Estratificação do risco: $\mathrm{CHADS}_{2}$} \\
\hline Factores de risco & Pontuação \\
\hline $\mathrm{C}$ (Congestive) ICC recente & 1 \\
\hline $\mathrm{H}$ (Hypertension) HTA & 1 \\
\hline $\mathrm{A}$ (Age) Idade $>75$ anos & 1 \\
\hline $\mathrm{D}$ (Diabetes mellitus) Diabetes & 1 \\
\hline $\mathrm{S}_{2}$ (Stroke) Historia de AVC ou AIT & 2 \\
\hline
\end{tabular}

Risco elevado: >3; Risco intermédio: 2-3; Risco baixo: 0-1 Legenda: ICC: insuficiência cardíaca congestiva.

ser ainda recente, não foi validada a capacidade predictiva do eventos embólicos desta escala.

Perante a estratificação de risco, independentemente da escala usada (CHADS 2 ou NICE), face a um risco elevado, iniciar-se-ia anticoagulação, numa situação de risco baixo, iniciar-se-ia antiagregação e no risco intermédio, poder-se-ia optar por qualquer uma das estratégias.

Para além da avaliação dos benefícios do controlo da frequência cardíaca versus o controlo do ritmo em utentes com FA, os estudos AFFIRM e RACE analisaram também o risco de eventos trombo-embólicos. $\mathrm{O}$ achado mais importante foi que os utentes que apresentaram AVC isquémico tinham descontinuado a anti-coagulação ou estavam em níveis sub-terapêuticos (23 de 35 utentes $-66 \%$, no RACE e 113 de 157 utentes $-72 \%$, no AFFIRM). Um dado ainda mais relevante foi que os 75 $\%$ dos utentes do grupo de controlo do ritmo que experimentaram um AVC apresentavam ritmo sinusal. Esta informação sugere que a anticoagulação com um INR 2-3 deve ser continuada apesar de se controlar o ritmo cardíaco, reconsiderando-se a decisão de parar a anticoagulação nos utentes cardiovertidos e em ritmo sinusal.

$\mathrm{O}$ tratamento antiagregante utiliza-se na FA para prevenir acidentes tromboembólicos quando existe um risco baixo (ver Quadros II, III). Pode-se utilizar o ácido acetilsalicílico (AAS) ou clopidrogrel. Actualmente o uso da ticlopidina está muito limitado pela importância dos seus efeitos secundários que, embora não sendo muito frequentes, são muito graves (neutropenia, trombocitopenia, anemia aplásica e púrpura trombocítica trombopénica), pelo que requer uma estreita vigilância e controlos hematológicos, de maneira espe-

\begin{tabular}{l} 
QUADRO IV. Estratificação do risco do NICE \\
(Nacional Institute for Health and Clinical Excellence) \\
Alto risco \\
\hline Embolismo, AVC ou AIT prévio \\
Idade > 75 anos com HTA, DM ou doença vascular \\
Valvulopatia, IC ou diminuição da FE documentada \\
em ecocardiograma \\
Risco intermédio \\
$>$ ou igual a 65 anos SEM factores de risco \\
$<75$ anos COM factores de risco (DM, HTA, vasculopatia \\
periférica) \\
Baixo risco \\
$<65$ anos SEM factores de risco
\end{tabular}

Legenda: FE:Fracção de ejecção

cial durante o $1^{\circ}$ mês.

A dosagem de AAS na prevenção de acidentes tromboembólicos na FA é de 250-325 mg/dia. O efeito adverso mais relevante é a hemorragia, sendo o risco de hemorragia dose-dependente e geralmente ocorre com doses superiores a $325 \mathrm{mg} /$ dia. Esta hemorragia é mais frequente em utentes em que coexistem lesões gástricas ou a administração de anti-inflamatórios não esteróides (AINE). O clopidogrel é uma alternativa ao AAS, embora com um custo mais elevado. É importante assinalar que no estudo CAPRIE, ${ }^{19} \mathrm{em}$ doentes tratados com clopidrogel ou com o AAS, a incidência global de qualquer hemorragia foi 9,3\% em ambos os grupos. Nos doentes que receberam clopidogrel a taxa de ocorrência de hemorragia gástrica foi de $2,0 \%$ e nos doentes que receberam AAS as taxas foram de $2,7 \%(\mathrm{p}=0,05)$.

Antes de iniciar uma terapêutica antiagregante, $o$ médico de família tem que ter presente que a maioria dos doentes é candidata a AAS e poucos terão indicação para o clopidogrel. Pode ser necessário rever se utentes medicados em internamento com clopidogrel têm indicação para tal.

Assim, embora existam múltiplos esquemas de início, o mais recomendável é iniciar com varfarina 1,25 $\mathrm{mg}\left(1 / 4 \mathrm{cp}\right.$ ) (1 comprimido de Varfine ${ }^{\circledast}$ equivale a $5 \mathrm{mg}$ ) durante 3 dias, fazendo-se o controlo ao $4^{\circ}$ dia, que foi o que fizeram estes doentes.

No início da anticoagulação, pode haver um perío- 
do de utilização simultânea de varfarina e de heparina de baixo peso molecular (HBPM) subcutânea (por exemplo: Fraxiparina ${ }^{\circledR}$ Nadroparina 0,6 cada 12 horas). Este esquema é utilizado quando se pretende uma anticoagulação mais rápida e tem uma fundamentação biológica. A varfarina, nos primeiros dias de administração, tem uma actividade procoagulante. Isto porque ela inibe a formação não só de factores pró-coagulantes (factores II, VII, IX e X) mas também de inibidores da coagulação (proteínas $\mathrm{C}$ e S). Como a semi-vida dos factores pró-coagulantes é maior que dos anti-coagulantes, durante os primeiros dias de administração ainda existem em circulação factores pró-coagulantes, mas já não existem inibidores da coagulação. Esta tendência pró-coagulante pode ver-se agravada nos utentes com deficit congénito de proteína $\mathrm{C}$, desencadeando por vezes um quadro de coagulação intravascular disseminada. No entanto, ao nível dos Cuidados de Saúde Primários não é habitual efectuar este procedimento, já que as situações não são urgentes e essa anticoagulação se inicia só com anticoagulantes orais, realizando-se controlos com ajuste de dose, 2 ou 3 vezes por semana, durante 1-2 semanas, até que se estabilize o INR.

As contra-indicações absolutas ao uso da varfarina são a existência das seguintes patologias: demência, concentrações de creatinina sérica $>3 \mathrm{mg}$, anemia com $\mathrm{Hb}<10 \mathrm{~g} / \mathrm{dL}$, pressão arterial $>180 / 100 \mathrm{mmHg}$, alcoolismo crónico, história de hemorragias por consumo de varfarina, predisposição a (traumas) traumatismos cranianos ou que requeiram a administração contínua de antiinflamatorios não esteróides (AINE). Outro aspecto importante a ter em conta, e que foram objecto de um trabalho de revisão publicado neste número da RPCG, ${ }^{20}$ são as interacções medicamentosas e dos alimentos com a varfarina.

Algumas situações que se podem apresentar com alguma frequência ao Médico de Família e em que tem que se tomar em consideração a anticoagulação são: extracção dentária, uma pequena cirurgia ou introdução de um novo fármaco ao utente.

No caso da extracção dentária, a evidência disponível é escassa. No entanto, recomenda-se manter o tratamento com a anticoagulação oral, realizar um INR prévio de controlo e, depois da extracção, tratamento local com ácido aminocapróico e fazer compressão activa com um penso embebido neste fármaco.
No caso de uma pequena cirurgia:

- Dia -2: Não tomar anticoagulante oral (AO)

- Dia -1: Não tomar AO. HBPM 0,3 ml, s.c, pela tarde

- Dia 0: Cirurgia. Não administrar AO. HBPM, 0,3 ml às 24 horas da anterior

- Dia 1: AO à dose habitual no jantar.HBPM, 0,3 ml

- Dia 2 ao 5: AO e HBPM até que o INR normalize.21

\section{DISCUSSÃO DOS CASOS}

Nestes dois casos clínicos trata-se de uma FA permanente, já que não existe possibilidade de reversão ao ritmo sinusal e a sua manutenção. Assim, a nossa estratégia terapêutica para estes dois doentes focaliza-se no controlo da resposta ventricular e na profilaxia de fenómenos trombo-embólicos, baseando-nos na literatura acima mencionada.

No Centro de Saúde de Portalegre está disponível o «Coag Check» para o controlo do INR, contando com o apoio da enfermeira da equipa. Para uma melhor organização no local de trabalho, cada médico procurou marcar um dia concreto da semana os seus utentes anticoagulados. Foi instituído o programa informático Tao Net, pioneiro na Unidade Local de Saúde do Norte Alentejano. Este programa facilita o controlo dos doentes anticoagulados mediante um controlo automático da dose sugerida, segundo o valor do INR. É impressa uma folha para o utente com a posologia diária e a data do próximo controlo. Assim, a maioria dos utentes que eram controlados no Serviço de Hematologia no Hospital passou para os respectivos Centros de Saúde, com a consequente poupança económica e de tempo.

No primeiro caso clínico, refere-se que o utente esteve sem ir a consulta durante vários anos. Isto deveu-se ao facto do utente não ter um médico atribuído. Durante este período não fez nenhuma consulta hospitalar.

Nas primeiras consultas veio acompanhado por uma filha, com escolaridade universitária, o que facilitou a explicação e instauração da estratégia terapêutica. O utente estava a fazer terapêutica com captopril, diltiazem, amiodarona e ácido acetilsalicílico. Tal como foi referido anteriormente, não havia justificação para manter a amiodarona, pelo que esta foi retirada, mantendo-se o diltiazem e o captopril. Com o objectivo de uma maior comodidade posológica e facilitar o cumprimento da terapêutica, foi proposto ao utente substituir o captopril, que estava a fazer em 3 tomas/dia, por 
um IECA com uma semi-vida mais longa, para poder fazer uma única toma diária, mas o utente preferiu manter o captopril. A mudança de um medicamento para outro, mesmo que pressuponha uma maior comodidade posológica, é com frequência encarada com relutância, nomeadamente pelos indivíduos idosos. Este aspecto deve ser tido em conta ao propor uma substituição e respeitar as preferências dos utentes, sempre que seja possível, promovendo uma boa adesão à terapêutica.

Também foi retirado o ácido acetilsalicílico e iniciou-se a anticoagulação. A prevenção dos acidentes trombo-embólicos vasculares cerebrais é um dos objectivos da FA. No entanto, um erro frequente é a não administração de anticoagulantes orais, embora esteja indicada. No caso deste utente, existiam vários critérios que faziam aumentar o risco trombo-embólico. Aplicando a classificação de $\mathrm{CHADS}_{2}$ tinha um valor de 2 pontos (episódio embólico prévio e hipertensão arterial), pelo que tinha um risco intermédio; no entanto, aplicando a estratificação NICE, tinha de alto risco. Assim tinha indicação para estar anticoagulado segundo as recomendações internacionais referidas. ${ }^{8,9}$

No segundo caso, o facto de o doente viver sozinho e ter uma escolaridade básica fez com que o médico de família tivesse algumas dúvidas a respeito do correcto cumprimento da terapêutica. Só o facto de ter um grande apoio familiar, pois uma filha morava junto a ele, fez com que, finalmente, o médico de família se decidisse a iniciar a hipocoagulação. $\mathrm{O}$ doente foi encorajado e falou-se com a filha sobre a necessidade de manter uma vigilância apertada do INR e outros aspectos como as interacções medicamentosas.

O doente estava a fazer digoxina e clopidogrel. A digoxina é um dos fármacos recomendados para o controlo da resposta ventricular. No entanto, a digoxina só pode ser considerada como tratamento inicial em monoterapia em doentes predominantemente sedentários, o que não era o caso do utente. Os beta-bloqueantes e antagonistas do cálcio inotrópicos negativos devem ser o tratamento inicial em monoterapia em todos os utentes, excepto nos sedentários. No caso actual havia um bom controlo da frequência ventricular. $\mathrm{O}$ facto de o doente apresentar um tremor essencial, bastante limitativo do dia-a-dia, e não apresentar uma indicação específica para a toma da digoxina, levou à subs- tituição da terapêutica pelo propanolol. Iniciou-se com uma dose de $40 \mathrm{mg} / \mathrm{dia}$, que posteriormente se aumentou para a $80 \mathrm{mg} /$ dia, na forma de libertação prolongada. O tratamento de primeira eleição no tremor essencial é o propanolol, sendo, também, um dos fármacos recomendados para o controlo da resposta ventricular. O resultado foi uma melhoria significativa do tremor essencial, assim como um bom controlo da frequência ventricular.

Aplicando, quer a classificação de $\mathrm{CHADS}_{2}$, quer a escala de NICE, este utente teria risco intermédio e poder-se-ia ou antiagregar ou anticoagular. Atendendo à sua idade ser de 75 anos, decidiu-se iniciar a anticoagulação e retirar o clopidogrel, segundo as recomendações internacionais referidas. ${ }^{8,9} \mathrm{De}$ acordo com estas, os doentes de risco trombo-embólico moderado/elevado beneficiam com a anti-coagulação crónica com doses de varfarina ajustadas para atingir um INR de 2.0 a 3.0, excepto se apresentarem alguma contra-indicação específica, o que não era o caso deste doente.

É importante salientar o papel de transversalidade dos cuidados prestados pelo médico de família. Nestes dois casos apresentados, o motivo que leva aos utentes à consulta não tem relação com o problema da «fibrilhação auricular». É, assim, muito importante que, nas primeiras consultas, o médico de família leia com pormenor o processo clínico, faça uma boa anamnese e tenha presente os antecedentes, lista de problemas, terapêutica prolongada e a estrutura familiar

Sugerem-se algumas conclusões que podem ser úteis na prática do médico de família:

1. Considerar se está a fazer a terapêutica correcta, sabendo que como primeiro objectivo na FA permanente está o controlo da frequência ventricular e que os fármacos recomendados são os beta-bloqueadores, os antagonistas do cálcio com efeito inotrópico negativo (diltiazem e verapamil) e a digoxina. Este último deve ser usado como fármaco de $2^{\mathrm{a}}$ linha, caso exista contra-indicação para o uso dos primeiros. Não se justifica o uso da amiodarona como $1^{\mathrm{a}}$ linha e, sobretudo, durante anos e sem controlo dos efeitos secundários.

2. Vencer a resistência a iniciar a hipocoagulação, quando existe critério para isso. Pode-se utilizar a estratificação numérica $\mathrm{CHADS}_{2}$ e a escala NICE de forma complementar para avaliar o risco de acidente trom- 
bo-embólicos. Se o médico de família tem dificuldade em iniciar a anticoagulação, deve referenciar a um Serviço de Hematologia. Posteriormente, poderá considerar a opção de fazer ele próprio o controlo do INR. A ideia fundamental é nunca deixar de anticoagular um doente que tem indicação formal para isso.

3. Ter a certeza que o doente compreende os cuidados necessários com a hipocoagulação antes de a iniciar, nomeadamente vigilância apertada do INR com controlos periódicos, interacções medicamentosas e educação em termos de alimentação. Neste aspecto devemos considerar que ter apoio familiar pode ser uma importante ajuda, em alguns utentes, para um correcto cumprimento da terapêutica, evitando enganos.

\section{REFERÊNCIAS BIBLIOGRÁFICAS}

1. FeinbergWM, Blackshear JL, Lapaucis A, Kronmal R, Hart RG. Prevalence, age distribution, and gender of patients with atrial fibrillation: analysis and implications. Arch Intern Med 1995 Mar 13; 155 (5): 460-73.

2. Go AS, Hylek EM, Phillips KA, ChangY, Henault LE, Selby JV, et al. Prevalence of diagnosed atrial fibrillation in adults: national implications for rhythm management and stroke prevention: the AnTicoagulation and Risk Factors in Atrial Fibrillation (ATRIA) Study. JAMA 2001 May 9; 285 (18): 2370-5.

3. Fuster V, Rydén LE, Asinger RW, Cannom DS, Crijns HJ, Frye RL, et al. ACC/AHA/ESC guidelines for the management of patients with atrial fibrillation: executive summary. J Am Coll Cardiol 2001 Oct; 38 (4): 1231-66.

4. Psaty BM, Manolio TA, Kuller RW, Kronmal RA, Crushman M, Fried LP, et al. Incidence of and risk factor for atrial fibrillation in older adults. Circulation 1997 Oct 7; 96 (7): 2455-61.

5. Lévy S, Camm AJ, Saksena S, Aliot E, Breithardt G, Crijns HJ, et al. International consensus on nomenclature and classification of atrial fibrillation. J Cardiovasc Electrophysiol 2003 Apr; 14 (4): 443-5.

6. Myse D, Waldo A, DiMarco J, Domanski MJ, Rosenberg Y, Schron EB, et al; Atrial Fibrillation Follow up Investigation of Rhythm Management (AFFIRM) Investigators. A comparison of rate control and rhythm control in patients with atrial fibrillacion. N Engl J Med 2002 Dec 5;347(23):1825-33.

7. Hagens VE, Crijns HJ, Van Veldhuisen DJ, Van Den Berg MP, Rienstra M, Ran chhor AV, et al.: Rate control versus rhythm control for patients with persistent atrial fibrillation with mild to moderate heart failure: results from the Rate Control versus Electrical cardioversion (RACE) study. Am Heart J 2005 Jun; 149 (6): 1106-11.

8. Snow V, Weiss KB, LeFevre M, McNamara R, Bass E, Green LA, et al. Management of newly detected atrial fibrillation: a clinical practice guideline from American Academy of Family Physicians and the American College of Physicans. Ann Intern Med 2003 Dec 16; 139 (12): 1009-17.

9. Fuster V, Rydén LE, Asinger RW, Cannom DS, Crijns HJ, Frye RL, et al. ACC/AHA/ESC guidelines for the management of patients with atrial fibrillation: a report of the American College of Cardiology/American Heart Association Task Force on Practice Guidelines and the European
Society of Cardiology for Practice Guidelines and Policy Conferences. Eur Heart J 2001 Oct; 22 (20):1 852-923.

10. Alvarez de la Cadena-Sillas JA, Lepe Montoya L. Amiodarona: efectos colaterales en 200 pacientes mexicanos seguidos durante 2 años: estudio prospectivo. Rev Mex Cardiol 2002; 13 (4): 149-52.

11. Benavente $O$, Hart R, Koudstaal P, Laupacis A, McBride R. Antiplatelet therapy for preventing stroke in patients with non-valvular atrial fibrillation and no previous history of stroke or transient ischemic attacks. Cochrane Database Syst Rev 2000; (2): CD001925.

12. Van Walraven C, Hart RG, Singer DE, Laupacis A, Connolly S, Petersen P, et al. Oral anticoagulants vs aspirin in nonvalvular atrial fibrillation: an individual patient meta-analysis. JAMA 2002 Nov 20; 288 (19): 2441-8.

13. Atrial fibrillation investigators. Risk factors for stroke and efficacy of antithrombotic therapy in atrial fibrillation. Analysis of pooled data from five randomized controlled trials. Arch Intern Med 1994 Jul 11; 154 (13): 1449-57.

14. Hart RG, Pearce LA, McBride R, Rothbart RM, Asinger RW. Factors associated with ischemic stroke during aspirin therapy in atrial fibrillation: analysis of 2012 participants in the SPAF I-III clinical trials: The Stroke Prevention in Atrial Fibrillation (SPAF) investigators. Stroke 1999 Jun; 30 (6): 1223-9.

15. Gage BF, Waterman ÂD, Shannon W, Boechler M, Rich MW, Radford MJ. Validation of clinical classification schemes for predicting stroke: results from the National Registry of Atrial Fibrillation. JAMA 2001 Jun 13; 285 (22): 2864-70.

16. Rockson SG, Albers GW. Comparing the guidelines: anticoagulation therapy to optimize stroke prevention in patients with atrial fibrillation. J Am Coll Cardiol 2004 Mar 17; 43 (6): 929-35.

17. Aguilar MI, Hart R. Oral anticoagulants for preventing stroke in patients with non-valvular atrial fibrillation and no previous history of stroke or transient ischemic attacks. Cochrane Database Syst rev 2005 Jul 20; (3): CD001927.

18. National Institute for Health and Clinical Excellence. Atrial fibrillation: the management of atrial fibrillation. London: NICE; 2006.

19. CAPRIE Steering Committe. A randomised, blinded, trial of clopidogrel versus aspirin in patients at risk of ischaemic events(CAPRIE). Lancet 1996 Nov 16; 348 (9038): 1329-39.

20. Lima N. Varfarina: uma revisão baseada na evidência das interacções alimentares e medicamentosas. Rev Port Clin Geral 2008 Jul-Ago; 24 (4): 475-82.

21. Ansell J, Hirsh J, Dalen J, Bussey H, Anderson D, Poller L, et al. Managing oral anticoagulant therapy. Chest 2001 Jan; 119 (1 Suppl): 22S-38S.

\section{ENDEREÇO PARA CORRESPONDÊNCIA}

Daniel Francisco Serrano Collantes

C/Martín Cansado, $n^{\circ} 3-2^{\circ}$

06002 Badajoz - Espanha

E-mail: danielcollantes@hotmail.com

\section{AGRADECIMENTOS}

Agradeço ao Dr. João Vasconcelos, cardiologista do Hospital Espírito Santo de Évora e do Hospital Dr. José Maria Grande, de Portalegre, pelos seus comentários. Agradeço ainda à Dr. ${ }^{a}$ Catarina Simas e à Enf. ${ }^{a}$ Maria Manuel Correia, do Centro de Saúde de Portalegre, a leitura corrigida deste artigo. 


\section{ABSTRACT}

Atrial fibrillation (AF) is the most widespread cardiac arrhythmia in Family Medicine, affecting 1-2\% of the general population and $5 \%$ of the population over 65 years old. Patients with this type of cardiac arrhythmia have a greater risk of death and morbidity, namely due to cerebral hemorrhage. This article seeks to reflect on the international recommendations regarding AF therapy and the family physician's practice, mostly pertaining to anticoagulation.

Therefore, we present two clinical cases of patients with AF. In both situations, the cases consisted of first-time consultations with the author during which the suitability of the prescribed therapeutics was evaluated and adjustments were made to the AF therapeutics. We expose some literature data that were used to fundament the decisions made regarding these two patients. According to international norms, the control of the heart rate associated with chronic anti-coagulation is the most diffused strategy relating to patients with AF. When heart rate control is intended, the drugs that stand first in line are beta-blockers and calcium-channel blockers. Finally, due to the fact that oral anticoagulation is not a risk-exempted therapeutics, there are scales that establish group-risks for thromboembolic events. These scales may help in the decision-making process related with antithrombotic therapeutics.

Additionally, we reflect on the significance of the international recommendations regarding the specificity of Family Medicine. As a provider of comprehensive and long-term health-care, the family physician stands on an optimum advantage point to judge if the AF therapeutics achieves the best possible results throughout several years of treatment. On the other hand, knowing the patient and his context, the physician can prompt the family into maintaining control over a therapeutic that demands a tight watch.

Keywords: Atrial Fibrillation; Anticoagulants; Pharmacotherapeutics; Family Physicians. 